\title{
The Avoidance Responses of Daphnia magna to the Exposure of Organophosphorus Pesticides in an On-Line Biomonitoring System
}

\author{
Zong-Ming Ren $\cdot$ Zhi-Liang Li $\cdot$ Jin-Miao Zha $\cdot$ \\ Kai-feng Rao • Mei Ma - Zijian Wang • Rong-Shu Fu
}

Received: 18 January 2007 / Accepted: 18 December 2007 / Published online: 12 January 2008

(C) Springer Science + Business Media B.V. 2007

\begin{abstract}
In this study, avoidance behavior of the freshwater cladoceran Daphnia magna Straus was used as indicator to assess the early stress of accidental organophosphorus pesticide (OP) contamination. The movement behavior was detected by a multi-species biomonitoring system. There was obvious concentration-response relationship between the OP stress and the behavioral response even at sublethal exposure. A rising OP stress resulted in a significant decrease of response time to escape (RTE; $p<0.05$ ). In comparison of different OPs, it was found that there was a power regression between RTE and the toxic unit of OPs. Therefore, the avoidance behavior of D. magna was a sensitive indicator of sublethal OP stress, and the power relationship could be used to predict the early warning thresholds of more OPs in the on-line biomonitoring system.
\end{abstract}

Keywords Avoidance behavior Daphnia magna . Organophosphorus pesticide (OP) $\cdot$ Response time to escape (RTE) · Toxic unit (TU)

Z.-M. Ren · Z.-L. Li • J.-M. Zha $\cdot$ K.-f. Rao $\cdot$ M. Ma •

Z. Wang $(\triangle)$

State Key Laboratory of Environmental Aquatic Chemistry,

Research Center for Eco-Environmental Science,

Chinese Academy of Science,

Shuangqing Rd 18, Haidian District, P.O. Box 2871,

Beijing 100085, China

e-mail: wangzj@rcees.ac.cn

Z.-L. Li $\cdot$ R.-S. Fu

College of Life Science, Shandong Normal University,

Jinan 250014, China

\section{Introduction}

The widely used organophosphorus pesticides (OPs) exert their toxicity by inhibiting acetyl cholinesterase (AChE) of organisms $[3,6]$. Therefore, extensive use and discharges of OPs in environment may impair biological communities and the accidental release into source water may cause unexpected human intoxication.

The initial response of an individual to contaminant exposure is a possible change in its behavior due to visual or chemoreception (olfaction, taste) damage [22, 26]. Changes in the movement behavior of organisms can therefore be used as a suitable indicator in ecotoxicological risk assessment. According to previous research $[8,9$, $19,28]$, avoidance of contaminants is an adaptive behavior that may reduce exposure to harmful conditions, and failure to avoid exposure may result in reduced fitness and survival, eventually leading to detrimental effects. AChE inhibition may cause hyperactivity, loss of coordination, convulsions, paralysis, and other kinds of behavioral changes. Hence, it may be appropriate to assess OP toxicological impact by detecting the avoidance behavioral changes in a non-target invertebrate species. Invertebrate behavioral studies are most commonly developed [2, 13, 16]. The freshwater cladoceran Daphnia magna Straus are normally considered as sensitive to contaminants [23, 29], and behavioral responses to contaminants were reported $[11,21,30]$.

The current study was undertaken to assess the effects of four OPs on the avoidance behavior of D. magna. The correspondence between the response time to escape (RTE) of D. magna and different OP stress, after 48-h exposure to dichlorvos, malathion, parathion, and methyl parathion, was investigated using the movement signal detected by a multi- 
species biomonitoring system to show whether the avoidance behavior was feasible as indicator to evaluate early stress of accidental OP contamination.

\section{Materials and Methods}

\subsection{Device}

The movement behavior of test organism in this study was detected by a multi-species biomonitoring system equipped with eight test chambers [10]. The measurement principle is based on quadropole impedance technique. Test organisms are placed in flow-through test chambers $(2 \mathrm{~cm}$ long, $1.5 \mathrm{~cm}$ in diameter) of the system, which is closed off on both sides with nylon nets $(250 \mu \mathrm{m})$. One pair of electrodes at the walls of the test chambers sends a high frequency signal of altering current, which is received by a second pair of non current-carrying electrodes. The on-line movement signal of test organisms is transformed by the A/D transformer. Translated by discrete Fast Fourier Transform, on-line movement signal is changed into time series data simultaneously, and the original data can be only exported in movement frequency type that changes from $0.5 \mathrm{~Hz}$ $(-0.5)$ to $8.5 \mathrm{~Hz}(-8.5)$. The signal changes formed by the $\mathrm{A} / \mathrm{D}$ transformer are analyzed automatically. The on-line monitoring data are analyzed using functions of "alarm generation," which is based on the alarm algorithm in an ARIMA-model.

\subsection{Test Species}

The experimental D. magna (24-h young) were obtained from the Chinese Environmental Science Academy and cultured in our laboratory for more than three generations. The general culture of D. magna was maintained in the standard reference water (SRW) [15], at $20 \pm 2^{\circ} \mathrm{C}$ with $16 \mathrm{~L} / 8 \mathrm{D}$ photoperiod. D. magna were fed with a suspension of batch-cultured green algae (Scenedesmus obliquus).

Before the exposure experiments, the gravid female $D$. magna were taken out and cultured individually in 50-ml glass beakers of SRW until they oviposited. As D. magna neonates from the first brood are usually the smallest [18] and much more sensitive to different growth media, healthy neonates (about $24 \mathrm{~h}$ ) were always taken from the second and following clutches as recommended [1]. During the individual culture of female $D$. magna with eggs, the growth medium was renewed regularly, and D. magna were fed with $S$. obliquus algae three times a week. Before feeding D. magna, the culture medium of $S$. obliquus was filtered and then diluted by SRW until the concentration reached $1 \times 10^{5}$ cells $/ \mathrm{ml}$. The quantity of the algae was about $1 \%$ beaker volume.

\subsection{Test Chemicals}

Dichlorvos, malathion, parathion, and methyl parathion were purchased from J\&K Chemical Ltd. All compounds were technical grade ( $>95 \%$ purity). Stock solutions (stored at $4^{\circ} \mathrm{C}$ until use) with proper concentration of each chemical were prepared in dimethyl sulfoxide (DMSO) with appropriate aliquots used to make each test solution of a specific concentration. All solvents were of analytical grade.

The concentration of DMSO in water was less than $0.5 \%$ in all experiments. A study has shown that DMSO of such concentration would neither lead to acute toxicity to $D$. magna nor affect the mobility of D. magna [25].

\subsection{Experimental Setup}

According to previous works $[4,12,17]$ and the Pesticide Ecotoxicity Database of US Environmental Protection Agency [31], the 48-h median lethal concentrations $\left(\mathrm{LC}_{50-48}\right)$ for D. magna (24 h young) were $0.20(0.14$ $0.27) \mu \mathrm{g} / \mathrm{l}$ for dichlorvos, $2.2(0.7-3.5) \mu \mathrm{g} / \mathrm{l}$ for malathion, $5.0(2.7-7.5) \mu \mathrm{g} / \mathrm{l}$ for parathion, and $8.7(6.4-11.0) \mu \mathrm{g} / \mathrm{l}$ for methyl parathion. In this study, $\mathrm{LC}_{50-48}$ of different $\mathrm{OP}$ was taken as one toxic unit (1TU) and acute 48-h toxicity tests under flow-through conditions were performed for seven concentration gradients, i.e., $0.1 \mathrm{TU}, 0.5 \mathrm{TU}, 1 \mathrm{TU}, 2 \mathrm{TU}$, $5 \mathrm{TU}, 10 \mathrm{TU}$, and 20TU, to evaluate the effects of different OPs on the avoidance behavior of D. magna. Control groups were tested in a solvent solution (SRW99.5\%+0.5\% DMSO). No food was added during the experiments.

Although the behavior strength had been applied to assess the effects of environment stressor on the behavior of some organisms $[5,14]$, there was not a precise concept yet. In this study, behavior strength is defined as the intensity of behavior parameters representing motility. It is an integrative reflection of all instantaneous motions, e.g., swimming velocity, behavioral frequency, and movement extent, and so on. Full behavior strength was regarded as $1 \mathrm{U}$.

Five healthy neonates about $24 \mathrm{~h}$ of $D$. magna were selected at random and placed in test chamber. Controlling the flow rate of each test chamber about $21 / \mathrm{h}$, it was proved to have no effect on the motility of D. magna [20]. The data were sampled automatically every $10 \mathrm{~min}$, and six data records in $1 \mathrm{~h}$ were used to calculate the average. The averages within 48-h exposure were used to assess the behavior strength changes. The experiment was repeated three times for each 48 -h exposure.

\subsection{Statistical Analyses}

The average values of the three repetitions of exposure experiments were presented. One-way analysis of variance of Statistical Package for the Social Sciences 10.0 software 
was performed to compare the effects of OP stress on the behavior strength of D. magna with the controls. Curve fitting was performed with Origin 7.0.

\section{Results and Discussion}

\subsection{The Effects of OP Stress on the Behavior Strength}

Figures 1, 2, 3, and 4 showed the effects of different OPs on the behavior strength of D. magna in 48-h exposure. Within the control group, no significant changes in behavior strength were detected. In the case of OP exposures, whether exposed in high or low concentrations OPs, the movements of $D$. magna changed in a similar manner. Comparison of behavioral responses of D. magna to different OPs with the results from 48-h exposure experiments indicated that there were obvious concentration-response relationships between OPs and the behavioral changes. Higher concentrations of the OPs resulted in a greater degree of the behavioral changes, as well as a shorter response time for the first behavioral response. One TU of OPs usually exceeded avoidance threshold concentrations and even in the sublethal concentrations of OPs (0.1TU), the behavioral changes were evident. These results advised that the behavior strength was time-dependent (http://www.skleac.ac.cn/Publications.asp).

Besides these changes, the behavioral responses of $D$. magna to different OPs in the same concentration levels were similar. All the behavioral changes showed in Figs. 1, 2,3 , and 4 were in accordance with the general adaptation of organisms in relationship to environment stress according to the description of Selye [27]. Shortly, upon continued exposure to OPs stress, no organism could be maintained continuously in a state of alarm. If the stress was so drastic

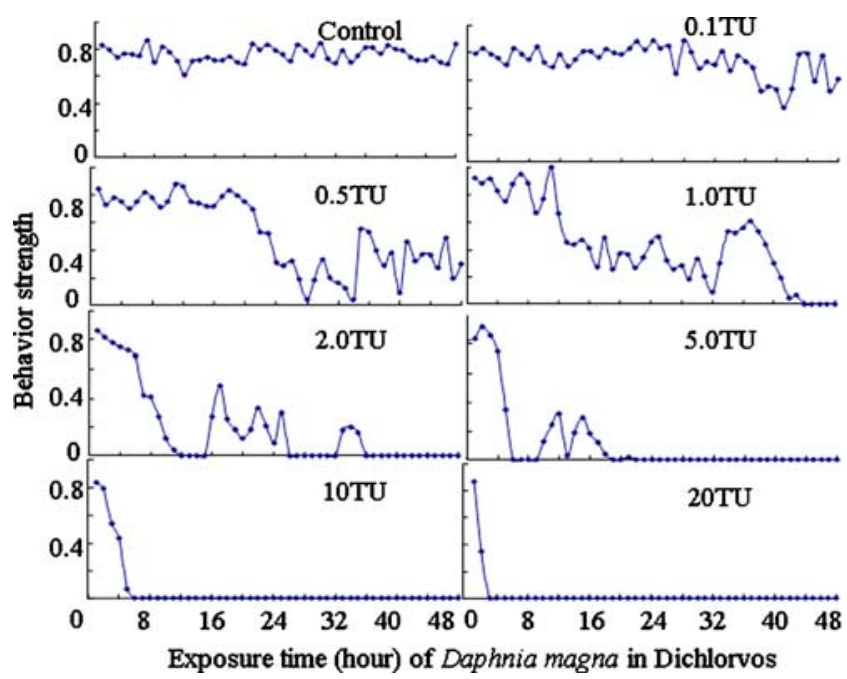

Fig. 1 The effects of Dichlorvos on the behavior strength of Daphnia magna in 48 -h exposure. ${ }^{*} P \leq 0.05$

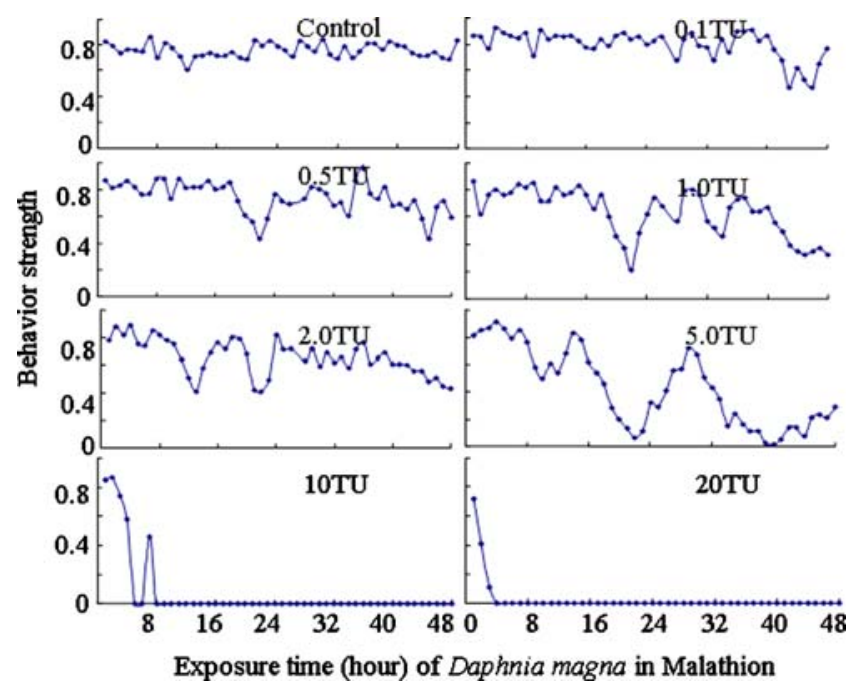

Fig. 2 The effects of Malathion on the behavior strength of Daphnia magna in 48 -h exposure. ${ }^{*} P \leq 0.05$

that continued exposure became incompatible with life, the organism lost its mobility during the alarm reaction (Threshold 1) within the first behavioral response phase in a short time (in 10TU and 20TU concentration levels of different OPs). If the organism could survive, this initial reaction was necessarily followed by a stage of resistance. After still more exposure to the stress (in 1TU, 2TU, and $5 \mathrm{TU}$ concentration levels of different OPs), the acquired adaptation is lost again (Threshold 2). The organism entered into a third phase, the stage of exhaustion. Under constant stress, the toxic effects happened. Once behavior adaptation had occurred, and ample energy was available, resistance based on the movement behavior should go on indefinitely (in $0.1 \mathrm{TU}$ concentration levels of different $\mathrm{OPs}$ ). The consequential reactions of D. magna in a certain behavior under OP stress included the categories of No effect, Stimulation, Acclimation, Adjustment, Toxic effect,

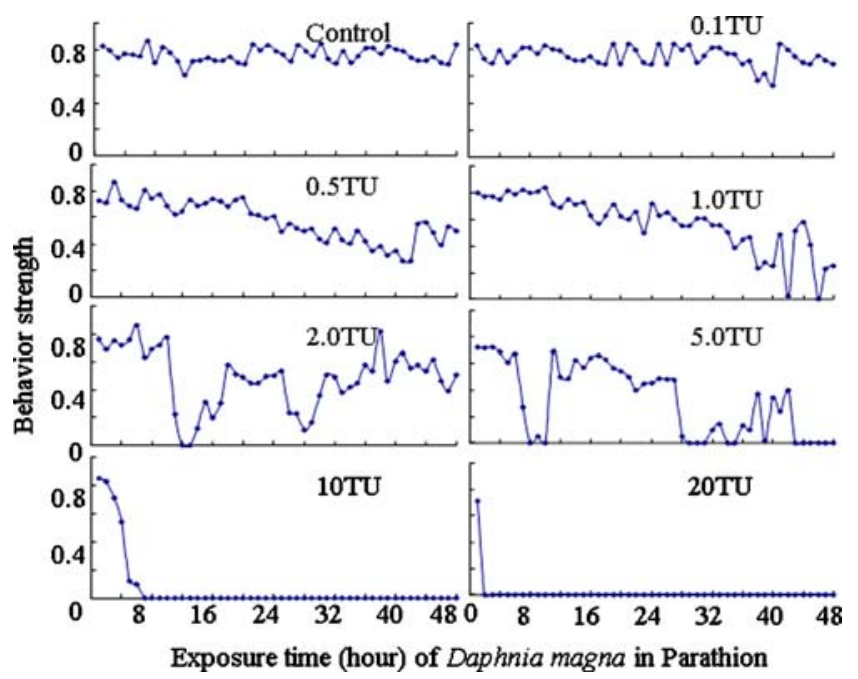

Fig. 3 The effects of Parathion on the behavior strength of Daphnia magna in 48 -h exposure. ${ }^{*} P \leq 0.05$ 


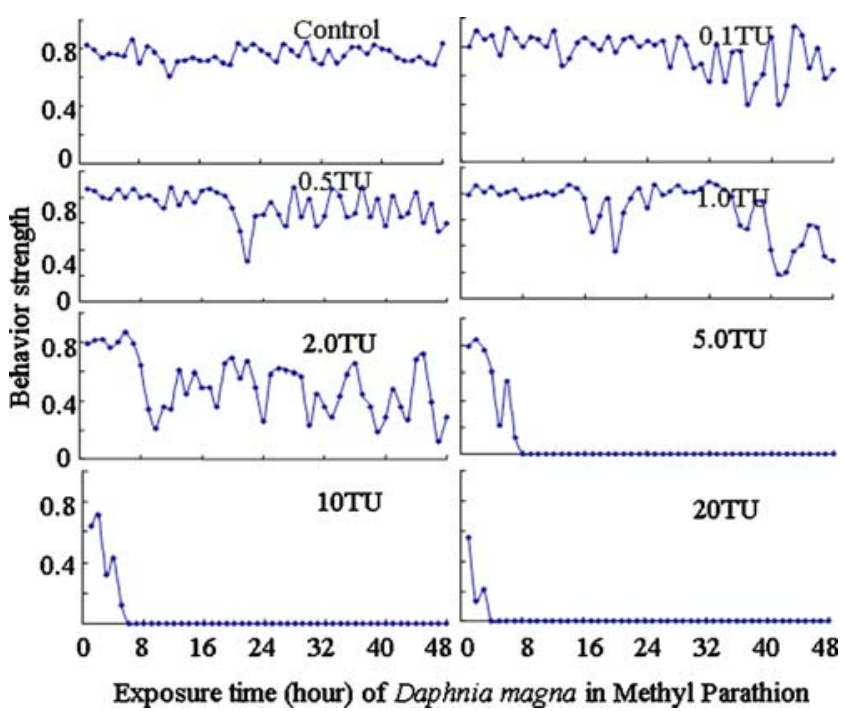

Fig. 4 The effects of Methyl Parathion on the behavior strength of Daphnia magna in 48 -h exposure. ${ }^{*} P \leq 0.05$

and Readjustment, as shown in Fig. 5. As the time length of different behavior categories was decided by OP stress, the lasting time of the time-dependent reactions of D. magna in a certain behavior under OP stress was highly variable, and it was normality if the lasting time of a certain behavior reactions was very short or even zeros (Figs. 1, 2, 3, and 4).

Different behaviors of different species had different toxicant sensitivity, but the first behavioral response to the stress, by which organisms tried to escape from the pollution source with higher behavior strength, was the general behavior adaptation of organisms, and it was defined as "avoidance behavior" [7, 8, 24]. Therefore, according to the results of the behavioral responses of D. magna to OP stress, avoidance behavior was mainly made up of no effects and stimulation actions in Fig. 5, and the RTE could be regarded as a feasible indicator in early warning of accidental OP contamination.

\subsection{The Relationships Between RTE and OP Stress}

Avoidance is an important defense mechanism in organisms, and no organism could maintain avoidance behavior at all

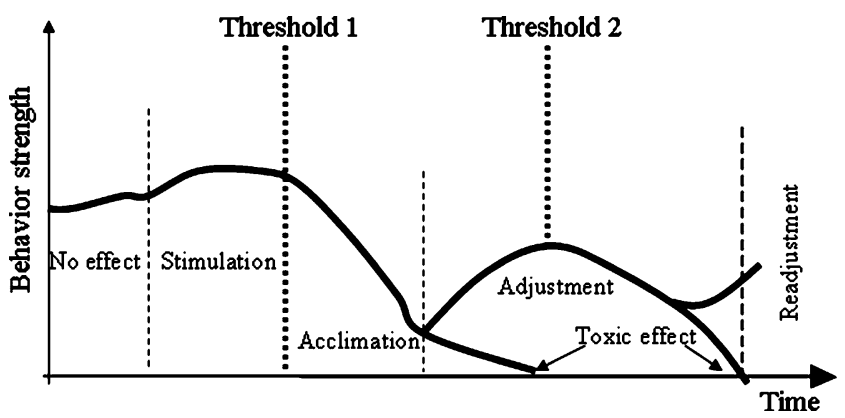

Fig. 5 The theoretical reactions of Daphnia magna in a certain behavior under OP stress times. In this study, once the decrease of behavior strength was significant in the first behavioral response of stress, it was regarded that the avoidance responses of test organisms to contaminations terminated. Therefore, RTE was determined by strength changes of the first behavioral response. And the first significant decrease of behavior strength was observed while differences between average values of first and second six data records were more than $20 \%$.

Figure 6 showed the changes of RTE in different OP stress. The relationship between RTE and the TU of OPs is defined as

$Y=12.12 X^{-0.70}$

$R^{2}=0.86$

where $Y$ represents RTE and $X$ represents the TU of OPs. The equation suggested that it was power relationship between RTE and TU of OPs, and a rising OP stress induced a significant decrease of RTE $(p<0.05)$.

According to the RTE Eq. 1, in the on-line monitoring accidental OP contamination, the measurement and alarm system were designed as follows.

Among the eight test chambers, two were used as control, three were used for test solutions, and the rest three were used for the 1:1 mixture of control and test solutions. Therefore, the relationships between RTE and OP stress could be described as

$\left\{\begin{array}{l}Y=12.12 X^{-0.70} \\ Y=12.12(X / 2)^{-0.70}-T\end{array}\right.$

where $T$ represents the difference of RTE between test solutions and 1:1 mixture of control and test solutions, and it could be showed directly by the system.

According to the RTE Eq. 2, if an accidental aquatic OP contamination happened, the biomonitoring system could detect the time when the accidental contamination happened ( $Y$ value) and the relative toxicity (based on TU) of OPs for test organisms ( $X$ value) in theory. Therefore, the

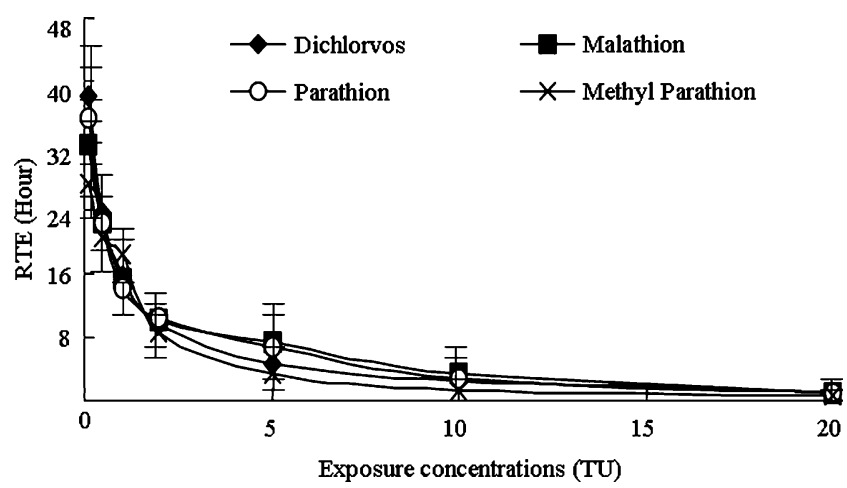

Fig. 6 The response time to escape (RTE) of Daphnia magna in different OP stress. Symbols Showed by mean \pm S.D. ${ }^{*} P \leq 0.05$ 
relationships between RTE and OP stress could be applied in early warning of accidental aquatic OP contamination.

\section{Conclusions}

This study proved behavioral responses of the freshwater cladoceran D. magna Straus to be sensitive bioindicators to OP stress. Changes in the behavioral parameter "behavior strength" can be used as early stress responses for acute aquatic OP contamination as part of ecological risk assessment.

The results from the present study showed that a rising OP stress induced a significant decrease of RTE $(p<0.05)$, which seems to be a very sensitive biological endpoint. An intense association was observed between the endpoint and avoidance; furthermore, avoidance was determined to be much more sensitive than lethality. Therefore, RTE should be recommended as a complementary tool in on-line monitoring accidental OP contamination.

Acknowledgments The work was supported by Natural Science Foundation of China (20621703, 20337020) and Natural Science Foundation of Beijing Municipality (8061004).

\section{References}

1. APHA. (2001). Standard methods for the examination of water and wastewater, 20th edition. American Public Health Association, American Water Works Association, and Water Pollution Control Federation, Washington, DC 20005.

2. Capowiez, Y., \& Berard, A. (2006). Assessment of the effects of imidacloprid on the behavior of two earthworm species (Aporrectodea nocturna and Allolobophora icterica) using $2 D$ terraria. Ecotoxicology and Environmental Safety, 64, 198-206.

3. Christopher, M. T., David, J. S., \& Matthew, J. W., (2001). Fluorinated phosphorus compounds: Part 4. A lack of anticholinesterase activity for four tris (fluoroalkyl) phosphates. Journal of Fluorine Chemistry, 107, 155-158.

4. Dortland, R. J. (1980). Toxicological evaluation of parathion and azinphosmethyl in freshwater model ecosystems. Agricultural Research Report, 898, 1-112.

5. Drastic, V., \& Kubečka, J. (2005). Fish avoidance of acoustic survey boat in shallow waters. Fisheries Research, 72, 219-228.

6. Duquesne, S. (2006). Effects of an organophosphate on Daphnia magna at suborganismal and organismal levels: Implications for population dynamics. Ecotoxicology and Environmental Safety, $65,145-150$.

7. Erik, H., Finn-Arne, W., Joachim, S., Svante, W., \& Holger, U. (2005). Avoidance behavior and brain monoamines in fish. Brain Research, 1032, 104-110.

8. Eriksson Wiklund, A.-K., Börjesson, T., \& Wiklund, S. J. (2006). Avoidance response of sediment living amphipods to zinc pyrithione as a measure of sediment toxicity. Marine Pollution Bulletin, 52, 96-99.

9. Farr, A. J., Chabot, C. C., \& Taylor, D. H. (1995). Behavioral avoidance of fluoranthene by fathead minnows (Pimephales promelas). Neurotoxicology and Teratology, 17, 265-271.
10. Gerhardt, A., Janssens, B., Mo, Z., Wang, C., Yang, M., \& Wang, Z. (2002). Short-term responses of Oryzias latipes (Pisces: Adrianichthyidae) and Macrobrachium nipponense (Crustacea: Palaemonidae) to municipal and pharmaceutical wastewater in Beijing, China: survival, behavior, biochemical biomarkers. Chemosphere, 47, 35-47.

11. Gray, S., \& Roff, D. A. (2000). Behaviour plasticity without learning: phenotypic and genetic variation of naive Daphnia in an ecological trade-off. Animal Behaviour, 59, 929-941.

12. Guilhermino, L., Lopes, M. C., Carvalho, A. P., \& Soares, A. M. V. M. (1996). Inhibition of acetylcholinesterase activity as effect criterion in acute tests with juvenile Daphnia magna. Chemosphere, 32, 727738 .

13. Heupel, K. (2002). Avoidance response of different collembolan species to Betanal. European Journal of Soil Biology, 38, 273-276.

14. Hofmann, H. A., \& Schildberger, K. (2001). Assessment of strength and willingness to fight during aggressive encounters in crickets. Animal Behavior, 62, 337-348.

15. ISO. (1996). Water Quality-Determination of the acute lethal toxicity of substances to a freshwater fish [Brachdanio rerio (Hamilton-Buchanan), Teleostei, Cyprinidae]_Part 3: Flowthrough method. ISO/ DIS 7346/3.

16. Jury, S. H., Kinnison, M. T., Huntting, H. W., \& Watson, W. H. (1994). The behavior of lobsters in response to reduced salinity. Journal of Experimental Marine Biology and Ecology, 180, 23-37.

17. Kikuchi, M., Sasaki, Y., \& Wakabayashi, M. (2000). Screening of organophosphate insecticide pollution in water using Daphnia magna. Ecotoxicology and Environmental Safety, 47, 239-245.

18. Lampert, W. (1993). Phenotypic plasticity of the size at first reproduction in Daphnia: The importance of material size. Ecology, 74, 1455-1466.

19. Maltby, L., Kedwards, T. J., Forbes, V. E., Grasman, K., Kammenga, J. E., Munns, W. R., et al. (2001). Linking individual-level responses and population-level consequences. In D. J. Baird \& G. A. Burton Jr. (Eds.) Ecological variability: Separating natural from anthropogenic causes of ecosystem impairment (pp. 27-82). Pensacola, FL, USA: SETAC.

20. Ren, Z. M., Ma, M., \& Wang, Z. J. (2006). On-line biomonitoring of accidental drinking water organophosphorous pesticides contamination. Water \& Wastewater Engineering, 32, 17-20.

21. Reynaldi, S., Duquesne, S., Jung, K., \& Liess, M. (2006). Linking feeding activity and maturation of Daphnia magna following short-term exposure to fenvalerate. Environmental Toxicology and Chemistry, 25, 1826-1830.

22. Riddell, D. J., Culp, J. M., \& Baird, D. J. (2005). Behavioral responses to sublethal cadmium exposure within an experimental aquatic food web. Environmental Toxicology and Chemistry, 24, 431-441.

23. Rosa, E., Barata, C., Damasio, J., Bosch, M. P., \& Guerrero, A. (2006). Aquatic ecotoxicity of a pheromonal antagonist in Daphnia magna and Desmodesmus subspicatus. Aquatic Toxicology, 79, 296-303.

24. Sager, D. R., Hocutt, C. H., \& Stauffer Jr., J. R. (2000). Avoidance behavior of Morone americana, Leiostomus xanthurus and Brevoortia tyrannus to strobe light as a method of impingement mitigation. Environmental Science \& Policy, 3, 393-403.

25. Sandbacka, M., Christianson, I., \& Isomaa, B. (2000). The acute toxicity of surfactants on fish cells, Daphnia magna and fish-A comparative study. Toxicology in Vitro, 14, 61-68.

26. Scarfe, A. D., Jones, K. A., Steele, C. W., Kleerekoper, H., \& Corbett, M. (1983). Locomotor behavior of four marine teleosts in response to sublethal copper exposure. Aquatic Toxicology, 2, 335-353.

27. Selye, H. (1973). The evolution of the stress concept. American Scientist, 61, 692-699. 
28. Steele, C. W., Strickler-Shaw, S., \& Taylor, D. H. (1989). Behavior of tadpoles of the bullfrog, Rana catesbeiana, in response to sublethal lead exposure. Aquatic Toxicology, 14, 331-343.

29. Sturm, A., \& Hansen, P. D. (1999). Altered cholinesterase and monooxygenase levels in Daphnia magna and Chironomus riparius exposed to environmental pollutants. Ecotoxicology and Environmental Safety, 42, 9-15.
30. Untersteiner, H., Kahapka, J., \& Kaiser, H. (2003). Behavioral response of the cladoceran Daphnia magna STRAUS to sublethal Copper stress - validation by image analysis. Aquatic Toxicology, $65,435-442$.

31. USEPA. (2000). Pesticide Ecotoxicity Database [Formerly: Environmental Effects Database (EEDB)]. Office of Pesticide Programs. Environmental Fate and Effects Division, Washington, DC 20005. 